\title{
Prophylactic cranial irradiation or MRI surveillance for extensive stage small cell lung cancer
}

\author{
James M. Taylor ${ }^{1}$, Chad G. Rusthoven ${ }^{2}$, Drew Moghanaki ${ }^{3}$ \\ ${ }^{1}$ Department of Radiation Oncology, Sidney Kimmel Medical College \& Cancer Center at Thomas Jefferson University, Philadelphia, PA, USA; \\ ${ }^{2}$ Department of Radiation Oncology, University of Colorado-Denver, Aurora, CO, USA; ${ }^{3}$ Department of Radiation Oncology, Atlanta VA Health \\ Care System, Emory University School of Medicine, Atlanta, GA, USA \\ Contributions: (I) Conception and design: JM Taylor, D Moghanaki; (II) Administrative support: D Moghanaki; (III) Provision of study materials or \\ patients: JM Taylor; (IV) Collection and assembly of data: All authors; (V) Data analysis and interpretation: All authors; (VI) Manuscript writing: All \\ authors; (VII) Final approval of manuscript: All authors. \\ Correspondence to: Drew Moghanaki, MD, MPH. Department of Radiation Oncology, Emory University School of Medicine, Atlanta, GA, USA. \\ Email: drew.moghanaki@emory.edu.
}

\begin{abstract}
The treatment paradigm for extensive stage small cell lung cancer (ES-SCLC) is evolving. Prophylactic cranial irradiation (PCI) has long been considered a component of standard treatment in patients with extensive stage disease who respond to chemotherapy. However, in the modern era of magnetic resonance imaging, the role of PCI has become an area of controversy following conflicting level I evidence. Due to conflicting data and toxicity concerns, the routine use of PCI has declined. Recent improvements in systemic disease control with the use of immunotherapy and reductions in the toxicity attributable to PCI with hippocampal avoidance and memantine have reignited the discussion. As such, we present here a narrative review of PCI with a focus on historical milestones, randomized data, risk mitigation and future directions.
\end{abstract}

Keywords: Small cell lung cancer (SCLC); prophylactic cranial irradiation (PCI); MRI surveillance; extensive stage

Submitted Feb 21, 2020. Accepted for publication Mar 09, 2020.

doi: $10.21037 /$ jtd.2020.03.80

View this article at: http://dx.doi.org/10.21037/jtd.2020.03.80

\section{Introduction}

The optimal treatment strategy for patients with extensive stage small cell lung cancer (ES-SCLC) continues to evolve. Specifically, the role of prophylactic cranial irradiation (PCI) has become an area of controversy following the publications of two phase III randomized clinical trials in 2007 and 2017 that reported conflicting results in regard to the impact on overall survival (OS) $(1,2)$. Discussions about the potential benefit of PCI in ES-SCLC have been further complicated with the recent introduction of immune checkpoint inhibitors (ICI) that has now shown to improve disease and OS. The uncertainty that remains has led to the recent activation of a phase III randomized trial evaluating the role of PCI in the era of brain MRI surveillance and early salvage CNS-directed radiation therapy for both LS and ES-SCLC which will be discussed in this review within the context of historical milestones, randomized data, and current era of ICI.

\section{History of clinical research in ES-SCLC}

Small cell lung cancer (SCLC) accounts for approximately $15 \%$ of all newly diagnosed lung cancer cases and an estimated $60-70 \%$ of patients will be diagnosed with extensive stage disease at presentation $(3,4)$. As with many malignancies, the treatment paradigm for SCLC has evolved over time from surgical resection to combined modality therapy with chemoradiation (5). As treatment strategies developed, discoveries about the biology, patterns 
of spread and clinical presentation of SCLC led to the stratification of patients into limited and extensive stage disease in 1957 by the Veterans Administration Lung Cancer Study Group $(6,7)$. This stratification is generally made by radiation oncologists who determine whether or not all areas of visible disease can be encompassed within a radiation therapy portal (8). This distinction would be critical in driving the evolution of treatment paradigms for LS-SCLC and ES-SCLC over the following decades.

In 1969, data from the Veterans Administration hospitals demonstrated a benefit of radiotherapy over surgical resection for LS-SCLC (9) In that same year, single agent chemotherapy utilizing cyclophosphamide proved to have activity in SCLC and became one of the chemotherapies of choice for ES-SCLC (10). By the 1970's, studies identified that the combination of cisplatin and etoposide (EP) was highly active against SCLC and safer when given with whole brain radiotherapy or thoracic radiation, given that $\mathrm{EP}$ is not often associated with radiation recall $(5,11,12)$. This body of work eventually led to the use of combination EP as the standard of care in the treatment of ES-SCLC (13).

Despite advances in systemic control for LS-SCLC and ES-SCLC, intracranial failures remained common in both cohorts. Data then began to emerge that showed a benefit to PCI in patients with LS-SCLC by decreasing the rate of brain metastasis (14). Additional studies reported on the benefits of PCI in LS-SCLC which led to the widely cited systematic review by Aupérin et al. that found a 5.4\% improved rate of survival at three years whenever PCI was added to standard of care treatments for LS-SCLC (15).

Meanwhile, the treatment paradigm for ES-SCLC remained largely static for the next several decades. That is, until 2007 when the European Organization for Research and Treatment of Cancer (EORTC) reported a phase III randomized clinical trial of 268 patients that showed an OS benefit with the addition of PCI in ES-SCLC (1). In 2015 , a separate randomized phase III study of 498 patients conducted in the Netherlands and the United Kingdom (CREST trial) reported an OS benefit with the addition of consolidative thoracic radiotherapy in ES-SCLC in participants who responded to chemotherapy; both arms of the CREST trial received PCI (16). However, subsequent randomized trials were unable to confirm the findings from the EORTC and CREST trial.

The first non-confirmatory study was the phase II RTOG 0937 that attempted to further assess the role of consolidative radiotherapy in ES-SCLC with local treatment of up to four metastatic sites and included PCI in both treatment arms. It was closed in 2015 after 86 patients were randomized due to a futility analysis showing that it was unlikely there would be a survival advantage in the study (17). Next, a Japanese randomized trial challenged the benefit of PCI in the era of brain MRI surveillance and early salvage radiation therapy and demonstrated no significant difference in OS between MRI surveillance with and without PCI (2). The details of this latter trial and its implications are discussed in further detail below.

Meanwhile, following a lack of progress for decades with improvements in systemic therapy, the IMpower133 trial demonstrated an OS benefit for ES-SCLC when the ICI atezolizumab which was approved by the FDA in 2019 was added to etoposide plus a platinum chemotherapy (18). It deserves emphasis that this study did not mandate PCI and only $11 \%$ of participants received PCI. This is likely a reflection of changing paradigms and concerns for increased neurotoxicity attributable to PCI. Yet, with such low numbers and without any randomization of PCI in that trial, patients with ES-SCLC who receive ICI and are now living longer have an unknown benefit with PCI and may be at increased risk for developing neurological complications and premature death from uncontrolled brain metastases.

\section{Methods}

We performed a search of the PubMed database to identify pertinent articles relating to the treatment of ES-SCLC and PCI. In addition to database searches, hand searches were also conducted based on expert opinion and author consensus. Searches of published abstracts were also included in the formation of the manuscript when full articles were not available. Lastly, to ensure thoroughness of the search, ClinicalTrials.gov was queried and protocols of interest were included for discussion. Finally, the manuscript and reference list were evaluated and approved by all authors for inclusion in the current publication.

\section{Biologic plausibility}

A question that is often asked is "what was the original thinking behind the potential benefit with PCI in patients with ES-SCLC?”. The biological plausibility and premise with PCI in ES-SCLC stemmed from awareness of the extremely high rate of brain metastasis in this population, coupled with the understanding that the CNS serves as a sanctuary site for SCLC cells. Studies have estimated the 
Table 1 Evidence based guidelines for PCI in ES-SCLC

\begin{tabular}{lcl}
\hline Organization & Most recent year updated & Position on PCI for ES-SCLC \\
\hline European Society of Medical Oncology & 2013 & PCI recommended, Grade IA \\
American College of Chest Physicians & 2013 & PCI recommended, Grade IB \\
American Society of Clinical Oncology & 2015 & PCI recommended, Grade IB \\
National Comprehensive Cancer Network & 2020 & Consider PCI or MRI surveillance \\
American Society of Radiation Oncology & 2019 & Consider PCl or MRI surveillance \\
\hline
\end{tabular}

$\mathrm{PCl}$, prophylactic cranial irradiation; ES-SCLC, extensive stage small cell lung cancer.

incidence of brain metastases in ES-SCLC to approach 58\% at 2 years after initiation of chemotherapy, and it is often the primary cause of death (19). Additional data following all patients with SCLC estimates that approximately $75 \%$ of patients will develop brain metastasis regardless of treatment $(19,20)$. With uncontrolled disease within the CNS as a predominant cause of death, the hypothesis was developed that PCI in all patients could improve survival outcomes by reducing neurologic death (21). Randomized trials eventually demonstrated significant reductions in the incidence of brain metastasis; however, they initially failed to demonstrate improvements in OS (14). Eventually, a pair of meta-analyses demonstrated an OS benefit with PCI in patients with LS-SCLC who had a complete response to chemotherapy $(15,22)$. This contributed to an evidence base that supported later efforts to evaluate the benefit of PCI for ES-SCLC. It remained unclear, however, if PCI would offer improved CNS control solely by addressing subclinical microscopic disease in the brain at the time of PCI, or if there might be additional effects on the CNS microenvironment that make the brain a less receptive "soil" for metastatic seeding. Regardless, even if the benefits of PCI on CNS control primarily arose from addressing existing subclinical disease, it opened the doors to any strategy that might achieve the same goal, such as the development of systemic therapies with enhanced CNS activity and imaging advancements that allow for earlier detection and salvage CNS-directed therapies.

\section{Randomized data and controversies}

The first prospective randomized study to demonstrate a survival benefit to PCI in ES-SCLC was conducted by the EORTC and published in the New England Fournal of Medicine in 2007 (1). This trial randomized 286 patients with any response to systemic chemotherapy to PCI $v$ s. clinical observation. The primary outcome was time to symptomatic brain metastases while OS, toxicity and treatment costs were secondary outcomes. The authors reported that patients who received PCI had a lower risk of symptomatic brain metastases [HR 0.27; 95\% confidence interval (CI), 0.16-0.44] and lower cumulative incidence of brain metastases at 1 year compared with observation $14.6 \%$ vs. $40.4 \%$. The authors reported a two-fold increase in OS at 1-year PCI: $27.1 \%$ (95\% CI, 19.4-35.5) vs. $13.3 \%$ (95\% CI, 8.1-19.9). The study reported higher rates of hair loss and fatigue with PCI, although there were no statistically significant differences in cognitive functioning or emotional functioning. The authors concluded "prophylactic cranial irradiation should be part of standard care for all patients with small-cell lung cancer who have a response to initial chemotherapy, and it should be part of the standard treatment in future studies involving these patients". Before long, PCI become the standard of care for ES-SCLC in multiple evidence-based clinical practice guidelines (Table 1).

Although the findings were practice changing, the results of this EORTC trial were critiqued for not mandating any brain imaging either before or after 4-6 cycles of initial chemotherapy, or during an interval of no more than 5 weeks between the last cycle of chemotherapy and randomization. In addition, patients were not followed with routine imaging in either arm of the study, and brain imaging (either CT or MRI) was left to the discretion of the treating physician depending on clinical symptoms. While the omission of restaging brain imaging was standard of care practice at the time, it contributed to uncertainties about what percentage of patients may or may not have had any detectable brain metastases at the time of PCI. This is noteworthy as the incidence of brain metastases in SCLC at diagnosis has been reported to be as high as $25 \%$ with MRI staging, and a meaningful number of patients will develop brain metastases after first-line therapy and prior to 
Table 2 Summary of key prospective studies discussed

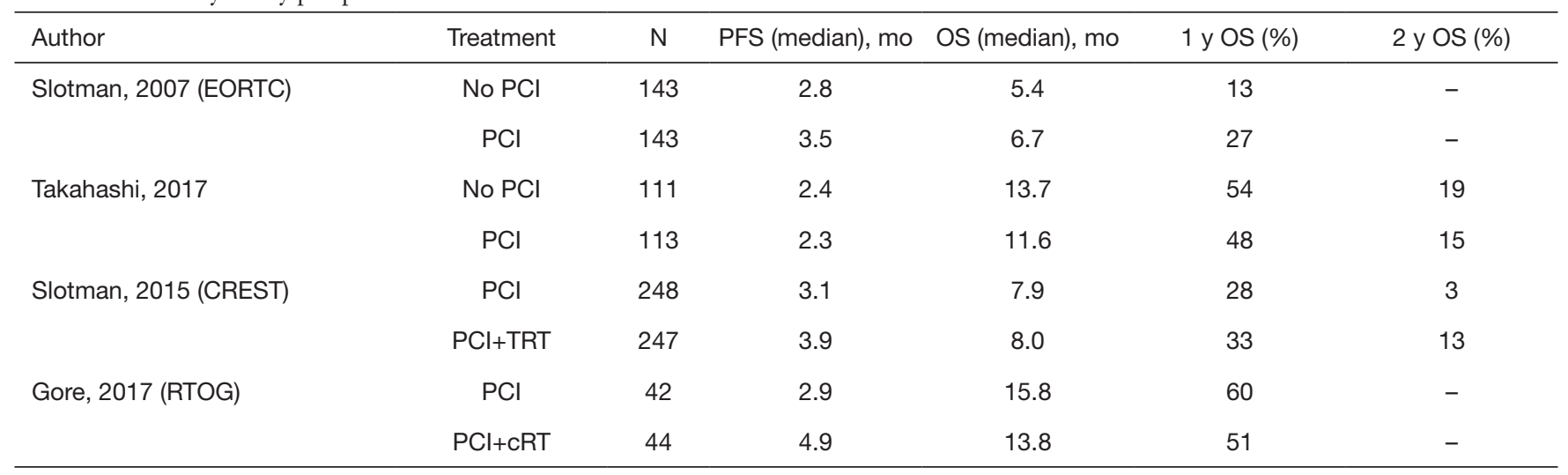

EORTC, European Organization for Research and Treatment of Cancer; RTOG, Radiation Therapy Oncology Group; CREST, Chest Radiotherapy Extensive Stage Trial; PCI, prophylactic cranial irradiation; TRT, thoracic radiotherapy; cRT, consolidative radiotherapy; PFS, progression free survival; OS, overall survival.

PCI $(23,24)$. Thus, a major concern was that the subset of patients with undetected brain metastases in the EORTC trial were randomized to actually receive therapeutic rather than PCI vs. observation until the time of neurologic symptoms related to their brain metastases. A recent systematic review and meta-analysis by Yin and colleagues in all patients with SCLC investigated this question by dichotomizing key studies of PCI into groups according to whether or not brain imaging was mandated (25). They found that PCI was only associated with a statistically significant OS benefit whenever studies did not mandate brain imaging (HR 0.81, 95\% CI, 0.67-0.99), but the OS benefit disappeared whenever studies mandated brain imaging (HR 0.94, 95\% CI, 0.74-1.18) (25).

The uncertainty of benefit with PCI for ES-SCLC that remained following this EORTC trial eventually led to a new randomized phase III trial by Takahashi et al. that required brain MRIs prior to enrollment to confirm the absence of brain metastases. This study, which was conducted in Japan, was an open-label trial that randomized 224 patients to MRI surveillance with and without PCI (2). Key inclusion criteria included any response to systemic chemotherapy and no evidence of brain metastases by MRI within 4 weeks of enrollment. All patients were then required to have routine brain imaging after randomization every 3 months for 1 year and again at 18 and 24 months. Although the earlier EORTC trial had already reported an OS advantage with PCI in ES-SCLC, the Japanese study design considered MRI surveillance alone the standard arm and used a one-sided alpha design to detect a $9 \%$ OS advantage at 2 years with the addition of PCI. The study was terminated early at a planned interim analysis due to futility (due to a $0.011 \%$ probability that PCI would improve OS over MRI surveillance alone) and the results were published in 2017. The authors reported a median OS of 11.6 (95\% CI, 9.5-13.3) months in the PCI group and 13.7 (95\% CI, 10.2-16.4) months in the observation group with a HR of 1.27 (95\% CI, 0.96-1.68, P=0.094). Thus, in the context of MRI surveillance, the Japanese trial did not confirm the OS benefit of PCI that was reported in the EORTC study despite similar decreases in brain metastases with PCI (see Table 2 for comparisons of key studies). The cumulative incidences of brain metastases at 6,12 , and 18 months were $15.0 \%$ (95\% CI, 9.2-22.3), 32.9\% (24.3$41.7)$, and $40.1 \%$ (31.0-49.1), in the PCI group and $46.2 \%$ (36.7-55.2), 59.0\% (49.1-67.6), and 63.8\% (54.0-72.1), in the observation group $(\mathrm{P}<0.0001)$, respectively. As in the EORTC trial, no differences in cognitive function were measured between groups. The authors of this Japanese study concluded that PCI in patients with ES-SCLC is not essential.

The conflicting data between the EORTC study and the Japanese data ultimately led to changes with recommendations in evidence-based clinical guidelines for ES-SCLC (see Table 1) (26). Key arguments about PCI for ES-SCLC have been centered around the impact of restaging and surveillance brain imaging with serial MRI scans. The Japanese trial suggested that in the context of close MRI surveillance and omission of upfront PCI, brain metastases that later occur may be effectively treated at time of detection without a decrement in OS. To this end, among patients in the MRI surveillance arm of the Japanese 
trial who developed brain metastases, $83 \%$ received salvage radiation. Conversely, among patients in the surveillance arm of the EORTC trial who developed symptomatic brain metastases, only $59 \%$ received salvage radiation. Thus, $41 \%$ with symptomatic brain metastases in the observation arm of the EORTC trial were unable to receive salvage radiation, raising the question of whether this difference could have contributed to the decreased OS without PCI. Other potential explanations for the differences in the effect of PCI may include biologic heterogeneity between the European and Japanese populations that has impacted randomized trial data before. For example, a study of irinotecan in LS-SCLC that was found to improve OS in a Japan Clinical Oncology Group randomized phase III trial could not be confirmed in a similar North American study $(27,28)$. Although, the similar magnitude of the reduction in brain metastases with PCI in the EORTC and the Japanese trial offers a meaningful counterargument to the hypothesis of heterogeneous population effects.

Finally, although non-randomized, a recently published single-institution retrospective study from Ottawa assessed the role of PCI in 397 patients with ES-SCLC without baseline brain metastases and who had a partial response to chemotherapy (29). In this report, Bang and colleagues identified a higher rate of OS in the 155 patients who received PCI compared to those who did not receive PCI (HR 0.55; 95\% CI, 0.39-0.77; $\mathrm{P}=0.0005$ ), even when MRI restaging was performed.

\section{$\mathrm{PCl}$ in the era of ICI}

While the aforementioned randomized trials of PCI and consolidative radiation therapy were ongoing for ESSCLC, multiple efforts had been underway in parallel to improve systemic disease control. Following multiple negative randomized trials, the phase III IMpower133 trial that was published in 2018 represented the first breakthrough in decades to show an improvement in OS for ES-SCLC (18). It randomized 403 patients to 4 cycles of induction etoposide plus platinum chemotherapy with either atezolizumab or a matching placebo, followed by maintenance atezolizumab or placebo and demonstrated a median OS benefit of 12.3 months (95\% CI, 10.8 to 15.9 ) in the atezolizumab arm compared with 10.3 months (95\% CI, 9.3 to 11.3 ) in the placebo arm. The use of PCI in that trial had not been mandated, was delivered to just over $11 \%$ of patients enrolled, and did not report the rates of brain metastases or neurological death. Another phase III trial of ICI in ES-SCLC was the CASPIAN trial which was published in 2019 and also showed an improvement in OS, this time with the addition of durvalumab (30). However, as with the Impower133 trial, the use of PCI in CASPAIN was poorly controlled, limited to patients in the chemotherapy arm, and delivered to only $8 \%$ of participants. The fundamental limitations with how PCI was utilized in these contemporary randomized trials introduces major limitations that obscure our understanding of how PCI in combination with ICI may help. On the one hand, the benefits of PCI to control CNS disease may become even more important with improved systemic disease control and prolonged overall survival with ICI. However, given ICIs have been found to penetrate the blood brain barrier and lead to objective responses in patients with known brain metastases, it is also possible that ICI may be sufficient to improve the control of microscopic CNS disease in a manner that dilutes previously seen benefits of PCI or even whole brain radiotherapy (31). Moreover, as prognoses improves, the benefits of avoiding early radiation to the entire brain in terms of cognitive function and QOL may increasingly favor a strategy of MRI surveillance and early salvage radiation therapy as needed. These hypotheses clearly need prospective investigations.

\section{Toxicity and risk mitigation of PCI}

Overall, the deleterious effects of PCI vs. observation on cognitive outcomes in patients with ES-SCLC have been poorly characterized. Initial data from earlier trials using insensitive screening metrics were unable to measure significant differences in cognitive outcomes between patients treated with PCI vs. observation (14,32). One explanation for this finding is that the competing risks of neurocognitive sequalae from systemic therapies and high rate of brain metastases in patients who do not receive PCI. Other studies of PCI have reported worse cognitive outcomes in patients treated with PCI using more sensitive screening metrics and neuroimaging (33-35). In a notable pooled analysis of the RTOG 0212 (lower vs. higher dose PCI for SCLC) and RTOG 0214 (PCI vs. observation for NSCLC) trials, PCI was associated with increased tested and self-reported cognitive toxicity at 6 and 12 months (32). However, it is critical to note that this analysis excluded patients who had developed brain metastases, potentially biasing the results in favor of the no-PCI cohort by censoring patients in the no-PCI cohort who may have experienced cognitive decline due to brain metastases that PCI is designed to prevent. 
The toxicity of PCI has now become more elucidated, owing to the contemporary use of patient reported outcome measures and the use of formal neuropsychological surveys in recent studies. Advances in brain imaging have also helped characterize distinct patterns that are associated with PCI and decline in neurocognitive function $(35,36)$.

As we look at the modern era of PCI for ES-SCLC, the questions that remain are bolstered by clinical advances that reduce the risks of neurocognitive toxicity associated with radiation through new strategies such as dose de-escalation, radioprotective pharmacological agents, and hippocampal avoidance treatment planning techniques. It has now been almost two decades since the randomized RTOG 0212 trial demonstrated decreased neurotoxicity with the use of $25 \mathrm{~Gy}$ instead of 36 Gy (37). The randomized phase III RTOG 0614 trial has since showed improved neurocognitive functioning with the use of memantine during whole brain radiation therapy, a drug that was initially approved by the FDA in 2003 that is believed to reduce the effects of excitotoxic glutamate release in the brain (38). And more recently, the randomized NRG CC001 trial of memantine and standard WBRT vs. WBRT with avoidance of the hippocampus, a limbic system structure critical to memory formation, demonstrated improved cognitive preservation and decreased symptoms interference in patients treated with hippocampal avoidance (39-41). The NRG CC003 is a phase II/III randomized trial of PCI in SCLC with and without hippocampal avoidance that is currently ongoing. The phase II portion of NRG CC003 was designed to determine whether the 12-month intracranial relapse with HA-PCI was non-inferior to standard PCI, and the ongoing phase III component is designed to determine whether HA-PCI can reduce the likelihood of 6-month cognitive deterioration on the Hopkins Verbal Learning Test-Revised (HVLT-R). Of note, prior smaller randomized trials of PCI $v s$. HA-PCI have returned conflicting results regarding the cognitive impact of HA-PCI $(42,43)$.

\section{New phase III trial and future directions}

As we move into a future for ES-SCLC that includes ICI, new studies are underway to better understand the role of PCI for both LS and ES-SCLC in the era of MRI surveillance and early salvage radiation therapy. This includes SWOG 1827, "MRI brain surveillance alone versus MRI surveillance and prophylactic cranial irradiation: a randomized phase III trial in small-cell lung cancer (MAVERICK)" which was activated in early 2020 with a planned enrollment of 600 patients with either LS-SCLC or ES-SCLC. The primary endpoint of the MAVERICK study tests the hypothesis that a strategy of MRI surveillance alone, allowing for early salvage radiation therapy in patients who develop brain metastases, can offer non-inferior OS when compared to MRI surveillance plus PCI. Key secondary endpoints will assess whether MRI surveillance alone is associated with improved cognitive preservation and quality of life, as well as the effect of PCI on OS in the subgroups with LS-SCLC or ES-SCLC. Prerandomization strata includes: (I) LS vs. ES disease, (II) planned ICI or not, and (III) performance status $0-1$ vs. 2. Importantly, stratification by ICI delivery should allow for rigorous analyses of the relative benefits and interaction of PCI and ICI. Given the importance of the PCI question to the majority of SCLC patients without brain metastases at diagnosis, MAVERICK is a pragmatic trial designed to minimize barriers to accrual allowing varying and evolving SCLC practice patterns including immunotherapy, consolidative thoracic radiation for ES-SCLC, HA-PCI, memantine, and salvage therapies including WBRT, HAWBRT, and SRS at the discretion of the treating physician. This trial is supported by a recent patterns of care survey amongst 487 radiation oncologists which reported that routine recommendation for PCI in ES-SCLC had dropped from $72 \%$ to $44 \%$ following the Japanese trial, and that $82 \%$ of respondents would be willing to enroll patients with either LS and/or ES SCLC on a trial comparing MRI surveillance with and without PCI (42-44). An important design feature of the MAVERICK trial is the prerandomization stratification by LS-SCLC and ES-SCLC which will ensure balance between the arms while allowing investigators to enroll the patients with the stage of SCLC for which they have equipoise on the PCI question (i.e., LS-SCLC, ES-SCLC, or both). The results from this trial will ultimately address many of the controversial questions that have generated equipoise regarding the role of PCI in ES-SCLC, and hopefully help define the role of PCI for LS-SCLC and ES-SCLC in the modern era of ICI and opportunity to manage with MRI surveillance and early salvage radiation therapy.

\section{Conclusions}

PCI clearly reduces the rates of brain metastases, but at potential costs to cognitive function and QOL. While landmark meta-analyses and randomized trials have demonstrated improved OS with PCI $(1,15)$ the benefit of 
PCI for ES-SCLC in the modern era of MRI surveillance and early salvage therapy was recently challenged by the Japanese randomized trial (2). Re-evaluating the role of PCI in the modern era is essential as various SCLC practice evolutions including routine MRI surveillance (45) emerging ICI agents, and growing interest in cognitive mitigation strategies including hippocampal-avoidance and radiosurgery can all shift the therapeutic ratio of PCI in the modern era (46-49). While the results of the phase III MAVERICK trial are awaited, it remains appropriate to consider PCI in patients with ES-SCLC on an individual basis after the risks and benefits are discussed including the need for adherence to routine MRI surveillance if PCI is omitted.

\section{Acknowledgments}

The authors would like to thank Drs. Ben Slotman and Andrew Turrisi for their thoughtful review and comments. Funding: None.

\section{Footnote}

Provenance and Peer review: This article was commissioned by the Guest Editor (Jose M. Pacheco) for the series "Small Cell Lung Cancer", published in Fournal of Thoracic Disease. This article has undergone external peer review.

Conflicts of Interest: All authors have completed the ICMJE uniform disclosure form (available at http://dx.doi. org/10.21037/jtd.2020.03.80). The series "Small Cell Lung Cancer" was commissioned by the editorial office without any funding or sponsorship. CGR reports that he is the national principle investigator of SWOG S1827/Maverick: MRI Brain Surveillance Alone versus MRI Surveillance and Prophylactic Cranial Irradiation (PCI): A Randomized Phase III Trial in Small-Cell Lung Cancer. The other authors have no other conflicts of interest to declare.

Ethical Statement: The authors are accountable for all aspects of the work in ensuring that questions related to the accuracy or integrity of any part of the work are appropriately investigated and resolved.

Open Access Statement: This is an Open Access article distributed in accordance with the Creative Commons Attribution-NonCommercial-NoDerivs 4.0 International License (CC BY-NC-ND 4.0), which permits the noncommercial replication and distribution of the article with the strict proviso that no changes or edits are made and the original work is properly cited (including links to both the formal publication through the relevant DOI and the license). See: https://creativecommons.org/licenses/by-nc-nd/4.0/.

\section{References}

1. Slotman B, Faivre-Finn C, Kramer G, et al. Prophylactic cranial irradiation in extensive small-cell lung cancer. $\mathrm{N}$ Engl J Med 2007;357:664-72.

2. Takahashi T, Yamanaka T, Seto T, et al. Prophylactic cranial irradiation versus observation in patients with extensive-disease small-cell lung cancer: a multicentre, randomised, open-label, phase 3 trial. Lancet Oncol 2017;18:663-71.

3. Torre LA, Bray F, Siegel RL, et al. A. Global cancer statistics, 2012. CA Cancer J Clin 2015;65:87-108.

4. Wang S, Zimmermann S, Parikh K, et al. Current Diagnosis and Management of Small-Cell Lung Cancer. Mayo Clin Proc 2019;94:1599-622.

5. Haddadin S, Perry MC. History of small-cell lung cancer. Clin Lung Cancer 2011;12:87-93.

6. Kallianos A, Rapti A, Zarogoulidis P, et al. Therapeutic procedure in small cell lung cancer. J Thorac Dis 2013;5 Suppl 4:S420-4.

7. Zelen M. Keynote address on biostatistics and data retrieval. Cancer Chemother Rep 3 1973;4:31-42.

8. Kalemkerian GP. Staging and imaging of small cell lung cancer. Cancer Imaging 2012;11:253-8.

9. Miller AB, Fox W, Tall R. Five-year follow-up of the Medical Research Council comparative trial of surgery and radiotherapy for the primary treatment of smallcelled or oat-celled carcinoma of the bronchus. Lancet 1969;2:501-5.

10. Green RA, Humphrey E, Close H, et al. Alkylating agents in bronchogenic carcinoma. Am J Med 1969;46:516-25.

11. Greco FA, Richardson RL, Snell JD, et al. Small cell lung cancer. Complete remission and improved survival. Am J Med 1979;66:625-30.

12. Sierocki JS, Hilaris BS, Hopfan S, et al. cisDichlorodiammineplatinum(II) and VP- 16-213: an active induction regimen for small cell carcinoma of the lung. Cancer Treat Rep 1979;63:1593-7.

13. Roth BJ, Johnson DH, Einhorn LH, et al. Randomized study of cyclophosphamide, doxorubicin, and vincristine versus etoposide and cisplatin versus alternation of these two regimens in extensive small-cell lung cancer: a phase III trial of the Southeastern Cancer Study Group. J Clin 
Oncol 1992;10:282-91.

14. Arriagada R, LeChevalier T, Borie F, et al. Prophylactic cranial irradiation for patients with small-cell lung cancer in complete remission. J Natl Cancer Inst 1995;87:183-90.

15. Aupérin A, Arriagada R, Pignon JP, et al. Prophylactic cranial irradiation for patients with small-cell lung cancer in complete remission. Prophylactic Cranial Irradiation Overview Collaborative Group. N Engl J Med 1999;341:476-84.

16. Slotman BJ, Van Tinteren H, Praag JO, et al. Use of thoracic radiotherapy for extensive stage small-cell lung cancer: A phase 3 randomised controlled trial. Lancet 2015;385:36-42.

17. Gore EM, Hu C, Sun AY, et al. Randomized Phase II Study Comparing Prophylactic Cranial Irradiation Alone to Prophylactic Cranial Irradiation and Consolidative Extracranial Irradiation for Extensive-Disease Small Cell Lung Cancer (ED SCLC): NRG Oncology RTOG 0937. J Thorac Oncol 2017;12:1561-70.

18. Horn L, Mansfield AS, Szcz sna A, et al. First-line atezolizumab plus chemotherapy in extensive-stage smallcell lung cancer. N Engl J Med 2018;379:2220-9.

19. Komaki R, Cox JD, Whitson Wisk of brain metastasis from small cell carcinoma of the lung related to length of survival and prophylactic irradiation. Cancer Treat Rep 1981;65:811-4.

20. Eagan RT, Frytak S, Lee RE, et al. A case for preplanned thoracic and prophylactic whole brain radiation therapy in limited small-cell lung cancer. Cancer Clin Trials 1981;4:261-6.

21. Lukas RV, Gondi V, Kamson DO, et al. State-of-the-art considerations in small cell lung cancer brain metastases. Oncotarget 2017;8:71223-33.

22. Meert AP, Paesmans M, Berghmans T, et al. Prophylactic cranial irradiation in small cell lung cancer: a systematic review of the literature with meta-analysis. BMC Cancer 2001;1:5.

23. Seute T, Leffers P, ten Velde GP, et al. Detection of brain metastases from small cell lung cancer: Consequences of changing imaging techniques (CT versus MRI). Cancer 2008;112:1827-34.

24. Manapov F, Klautke G, Fietkau R. Prevalence of brain metastases immediately before prophylactic cranial irradiation in limited disease small cell lung cancer patients with complete remission to chemoradiotherapy: A single institution experience. J Thorac Oncol 2008;3:652-5.

25. Yin X, Yan D, Qiu M, et al. Prophylactic cranial irradiation in small cell lung cancer: a systematic review and meta- analysis. BMC Cancer 2019;19:95

26. Slotman BJ. Time to reconsider prophylactic cranial irradiation in extensive-stage small-cell lung cancer? Lancet Oncol 2017;18:566-7.

27. Noda K, Nishiwaki Y, Kawahara M, et al. Irinotecan plus cisplatin compared with etoposide plus cisplatin for extensive small-cell lung cancer. N Engl J Med 2002;346:85-91.

28. Lara PN, Natale R, Crowley J, et al. Phase III trial of irinotecan/cisplatin compared with etoposide/cisplatin in extensive-stage small-cell lung cancer: Clinical and pharmacogenomic results from SWOG S0124. J Clin Oncol 2009;27:2530-5.

29. Bang A, Kendal WS, Laurie SA, et al. Prophylactic Cranial Irradiation in Extensive Stage Small Cell Lung Cancer: Outcomes at a Comprehensive Cancer Centre. Int J Radiat Oncol Biol Phys 2018;101:1133-40.

30. Paz-Ares L, Dvorkin M, Chen Y, et al. Durvalumab plus platinum-etoposide versus platinum-etoposide in firstline treatment of extensive-stage small-cell lung cancer (CASPIAN): a randomised, controlled, open-label, phase 3 trial. Lancet 2019;394:1929-39.

31. Kamath SD, Kumthekar PU. Immune Checkpoint Inhibitors for the Treatment of Central Nervous System (CNS) Metastatic Disease. Front Oncol 2018;8:414.

32. Gregor A, Cull A, Stephens RJ, et al. for Cancer UK. Prophylactic cranial irradiation is indicated following complete response to induction therapy in small cell lung cancer: results of a multicentre randomised trial. Eur J Cancer 1997;33:1752-8.

33. Gondi V, Paulus R, Bruner DW, et al. Decline in tested and self-reported cognitive functioning after prophylactic cranial irradiation for lung cancer: Pooled secondary analysis of radiation therapy oncology group randomized trials 0212 and 0214. Int J Radiat Oncol Biol Phys 2013;86:656-64.

34. Johnson BE, Becker B, Goff WB 2nd, et al: Neurologic, neuropsychologic, and computed cranial tomography scan abnormalities in 2- to 10-year survivors of small- cell lung cancer. J Clin Oncol 1985;3:1659-67.

35. Catane R, Schwade JG, Yarr I, et al: Follow-up neurological evaluation in patients with small cell lung carcinoma treated with prophylactic cranial irradiation and chemotherapy. Int J Radiat Oncol Biol Phys 1981;7:105-9.

36. Walker AJ, Ruzevick J, Malayeri AA, et al. Postradiation imaging changes in the CNS: How can we differentiate between treatment effect and disease progression? Future Oncol 2014;10:1277-97. 
37. Wolfson AH, Bae K, Komaki R, et al. Primary analysis of a phase II randomized trial radiation therapy Oncology Group (RTOG) 0212: Impact of different total doses and schedules of prophylactic cranial irradiation on chronic neurotoxicity and quality of life for patients with limiteddisease small-cell lung cancer. Int J Radiat Oncol Biol Phys 2011;81:77-84.

38. Brown PD, Pugh S, Laack NN, et al. Memantine for the prevention of cognitive dysfunction in patients receiving whole-brain radiotherapy: A randomized, double-blind, placebo-controlled trial. Neuro Oncol 2013;15:1429-37.

39. Gondi V, Pugh SL, Tome WA, et al. Preservation of memory with conformal avoidance of the hippocampal neural stem-cell compartment during whole-brain radiotherapy for brain metastases (RTOG 0933): A phase II multi-institutional trial. J Clin Oncol 2014;32:3810-6.

40. Gondi V, Deshmukh S, Brown PD, et al. NRG Oncology CC001: A phase III trial of hippocampal avoidance (HA) in addition to whole-brain radiotherapy (WBRT) plus memantine to preserve neurocognitive function (NCF) in patients with brain metastases (BM). J Clin Oncol 2019;37:2009.

41. Brown PD, Gondi V, Pugh S, et al. Hippocampal Avoidance During Whole-Brain Radiotherapy Plus Memantine for Patients With Brain Metastases: Phase III Trial NRG Oncology CC001. J Clin Oncol 2020;38:1019-29.

42. De Dios ND, Counago F, Lopez JL, et al. Phase III

Cite this article as: Taylor JM, Rusthoven CG, Moghanaki D. Prophylactic cranial irradiation or MRI surveillance for extensive stage small cell lung cancer. J Thorac Dis 2020;12(10):6225-6233. doi: 10.21037/jtd.2020.03.80
Trial of Prophylactic Cranial Irradiation with or without Hippocampal Avoidance for SMALL-CELL LUNG Cancer. Int J Radiat Oncol Biol Phys 2019;105:S35-36.

43. Belderbos J, Ruysscher D, Jaeger K, et al. OC-0503 phase III trial of prophylactic cranial irradiation with or without Hippocampus avoidance in SCLC. Radiother Oncol 2019;133:S259.

44. Gjyshi O, Ludmir EB, Pezzi TA, et al. Evolving Practice Patterns in the Use of Prophylactic Cranial Irradiation for Extensive-Stage Small Cell Lung Cancer. JAMA Netw Open 2019;2:e199135.

45. Kalemkerian GP, Akerley W, Bogner P, et al. Small cell lung cancer. J Natl Compr Canc Netw 2013;11:78-98.

46. Rusthoven CG, Kavanagh BD. Prophylactic cranial irradiation (PCI) versus active MRI surveillance for small cell lung cancer: the case for equipoise. J Thorac Oncol 2017;12:1746-54.

47. Robin TP, Jones BL, Amini A, et al. "Radiosurgery alone is associated with favorable outcomes for brain metastases from small-cell lung cancer." Lung Cancer2018;120:88-90.

48. Cifarelli CP, Vargo JA, Fang W, et al. Role of Gamma Knife Radiosurgery in Small Cell Lung Cancer: A MultiInstitutional Retrospective Study of the International Radiosurgery Research Foundation (IRRF). Neurosurgery 2020;87:664-71.

49. Rusthoven, Chad G. Small Cell Lung Cancer: PCI Uncertainty and Emerging Radiosurgery Interest. Int J Radiat Oncol Biol Phys 2019;103:1034-5. 